\title{
Shoulder and elbow motion during two speeds of wheelchair propulsion: a description using a local coordinate system
}

\author{
Michael L Boninger ${ }^{1,2}$, Rory A Cooper ${ }^{1,2}$, Sean D Shimada ${ }^{1,2}$ and Thomas E Rudy ${ }^{3}$ \\ ${ }^{1}$ Human Engineering Research Laboratories, Division of Physical Medicine and Rehabilitation, Department of \\ Orthopaedic Surgery, University of Pittsburgh; ${ }^{2}$ Department of Rehabilitation Science and Technology, School of \\ Health and Rehabilitation Sciences, University of Pittsburgh; ${ }^{3}$ Department of Anesthesiology and Biostatistics and \\ Pain and Evaluation and Treatment Institute, University of Pittsburgh, Pittsburgh, Pennsylvania, USA
}

\begin{abstract}
Individuals who propel wheelchairs have a high prevalence of upper extremity injuries. To better understand the mechanism behind these injuries this study investigates the motion of the shoulder and elbow during wheelchair propulsion. The objectives of this study are: (1) To describe the motion occurring at the shoulder and elbow in anatomical terms during wheelchair propulsion; (2) to obtain variables that characterize shoulder and elbow motion and are statistically stable; (3) to determine how these variables change with speed. The participants in the study were a convenience sample of Paralympic athletes who use manual wheelchairs for mobility and have unimpaired arm function. Each subject propelled an ultralight wheelchair on a dynamometer at 1.3 and 2.2 meters per second $(\mathrm{m} / \mathrm{s})$. Biomechanical data was obtained using a force and moment sensing pushrim and a motion analysis system. The main outcome measures investigated were: maximum and minimum angles while in contact with the pushrim, range of motion during the entire stroke and peak accelerations. All of the measures were found to be stable at both speeds (Cronbach's alpha >0.8). The following measures were found to differ with speed (data format: measure at $1.3 \mathrm{~m} / \mathrm{s} \pm \mathrm{SD}$; measure at $2.2 \mathrm{~m} / \mathrm{s} \pm \mathrm{SD})$ : minimum shoulder abduction angle during propulsion $\left(24.5^{\circ} \pm 6.7\right.$, $\left.21.6^{\circ} \pm 7.2\right)$, range of motion during the entire stroke in elbow flexion/extension $\left(54.0^{\circ} \pm 9.9\right.$, $\left.58.1^{\circ} \pm 10.4\right)$ and shoulder sagittal flexion/extension $\left(74.8^{\circ} \pm 9.4,82.6^{\circ} \pm 8.5\right)$, and peak acceleration in shoulder sagittal flexion/extension $\left(4044^{\circ} / \mathrm{s}^{2} \pm 946,7146^{\circ} / \mathrm{s}^{2} \pm 1705\right)$, abduction/adduction $\left(2678^{\circ} / \mathrm{s}^{2} \pm 767,4928^{\circ} / \mathrm{s}^{2} \pm 1311\right)$, and elbow flexion/extension $\left(9355^{\circ} / \mathrm{s}^{2} \pm 4120\right.$, $\left.12889^{\circ} / \mathrm{s}^{2} \pm 5572\right)$. This study described the motion occurring at the shoulder and elbow using a local coordinate system. Stable parameters that characterize the propulsive stroke and differed with speed were found. In the future these same parameters may provide insight into the cause and prevention of shoulder and elbow injuries in manual wheelchair.
\end{abstract}

Keywords: shoulder; elbow; motion; wheelchair; wheelchair propulsion; range of motion

\section{Introduction}

By the nature of wheelchair propulsion, manual wheelchair users (MWUs) are essentially walking with their arms. The upper extremity, particularly the shoulder, is designed for freedom of movement not repetitive loading. Not surprisingly, MWUs are prone to shoulder injuries. Survey studies of individuals with paraplegia have reported an absolute prevalence of shoulder pain of between 31 and $73 \% .^{1-4}$ In addition

Correspondence: ML Boninger

This study was supported by: The National Institutes of Health NCMRR Clinical Investigator Award (PA-93-082 and 1 P01 HD33989-01) and The United States Department of Veterans Affairs, Rehabilitation Research and Development Service (B686RA, B869-RA, and B805-RA). to the survey studies, a number of investigators have used imaging studies to investigate the cause of shoulder pain. Bayley et al found rotator cuff tears in $65 \%$ of individuals with paraplegia and shoulder pain. ${ }^{5}$ Wylie found radiographic shoulder abnormalities in $32 \%$ of the MWUs 20 years post injury. ${ }^{6}$

While the shoulder is the most commonly sited area of pain a high prevalence of elbow pain is also seen. Sie reported elbow pain in $16 \%$ of MWUs. ${ }^{4}$ Sie further defined significant pain as that which required analgesia, occurred with two or more ADLs or required cessation of activity. Using this definition, the prevalence of all upper extremity pain complaints was $20 \% 5$ years post injury, and $46 \%$ from $15-19$ 
years post injury. In all of these studies, the authors felt that pain and radiographic abnormalities were, at least in part, related to overuse of the arm during wheelchair propulsion.

This study describes the motion at the shoulder and elbow during wheelchair propulsion. Although a number of researchers have investigated upper extremity kinematics during wheelchair propulsion, ${ }^{7-13}$ this study incorporates the following key features that add to its clinical and scientific utility:

(1) motion is described in three dimensional space and is referenced to an anatomically relevant local coordinate system,

(2) the mathematics necessary to perform this type of analysis are clearly presented,

(3) rigorous statistical analysis is applied to determine the reliability of measures used to describe motion It is our belief that studying wheelchair propulsion biomechanics, will provide insight into the cause of upper extremity injuries in MWUs. The use of a local coordinate system based on arm anatomy allows for description of kinematic data in anatomical terms. We hypothesize that using a local coordinate system will enable us to find stable parameters that describe shoulder and elbow kinematics during wheelchair propulsion. In addition, we hypothesize that these parameters will show statistically significant differences at different speeds of propulsion.

\section{Methods}

The data collection methods and subjects used in this study are the same as those used in a previous article on wrist biomechanics. ${ }^{14}$ Therefore, a brief description of the methods is presented.

\section{Subjects}

A convenience sample of six manual wheelchair users (MWUs) gave informed consent to participate in this study. The subjects consisted of athletes who participated in the 1994 United States Olympic Committee, Wheelchair Sports USA Paralympic training camp and had unimpaired upper extremity function. Traumatic spinal cord injury was the cause of disability in all but one of the athletes who had spina bifida. The athletes participated in a variety of Paralympic sports including: table tennis, weight training, swimming, shooting and wheelchair racing. The average age of the subjects was 27.5 years (range $22-34$ ).

\section{Measurement system}

Kinetic Kinetic data were obtained at $240 \mathrm{~Hz}$ using a force and torque sensing pushrim (Smart ${ }^{\text {Wheel }}$ ) designed, fabricated, calibrated, and tested by Cooper et al. ${ }^{15,16}$ For the purpose of this paper, the Smart ${ }^{\text {Wheel }}$ was only used for determining the beginning and ending of the propulsive stroke. A light-weight manual wheelchair (Quickie Designs, Inc., 2842 Business Park Ave., Fresno,
CA 93727-1328) with $0.41 \mathrm{~m}$ seat depth and $0.41 \mathrm{~m}$ seat width, a $5^{\circ}$ seat angle, and an $85^{\circ}$ seat-to-backrest angle was configured with a SMART ${ }^{\text {Wheel }}$ attached to its right side. The SMART ${ }^{\text {Wheel }}$ has a standard pushrim. The wheel camber for both rear wheels was set to zero.

Kinematic Kinematic data was collected at $60 \mathrm{~Hz}$ with a three camera motion analysis system (PEAK5) and low-pass filtered at $6 \mathrm{~Hz} .{ }^{17}$ The kinematic data were post processed by using linear interpolation to increase the frequency to $240 \mathrm{~Hz}$ for analysis. Kinematic and kinetic data collection were synchronized via a computer generated synchronization pulse. Reflective markers were placed over bony landmarks on the arm, shoulder, and hip. The markers used for this analysis are pictured in Figure 1. The markers placed on the wrist were $6 \mathrm{~mm}$ in diameter, all other markers were $13 \mathrm{~mm}$ in diameter.

\section{Data collection}

The wheelchair with each subject, in-turn, was aligned and secured over the rollers of a computer numerically controlled (CNC) dynamometer. ${ }^{18,19}$ During the test session, subjects pushed the wheelchair on the dynamometer for $5 \mathrm{~min}$ to become accommodated. Each subject propelled the wheelchair at $3 \mathrm{mph}$ for 2 min with the power output of the subject controlled by the dynamometer at 14 watts. The subjects were allowed a rest period and then propelled the wheelchair at $5 \mathrm{mph}$ for $2 \mathrm{~min}$ at 23 watts. Data was collected for the last $15 \mathrm{~s}$ of each test. Pushrim force and moment data were collected at $240 \mathrm{~Hz}$ with a digital

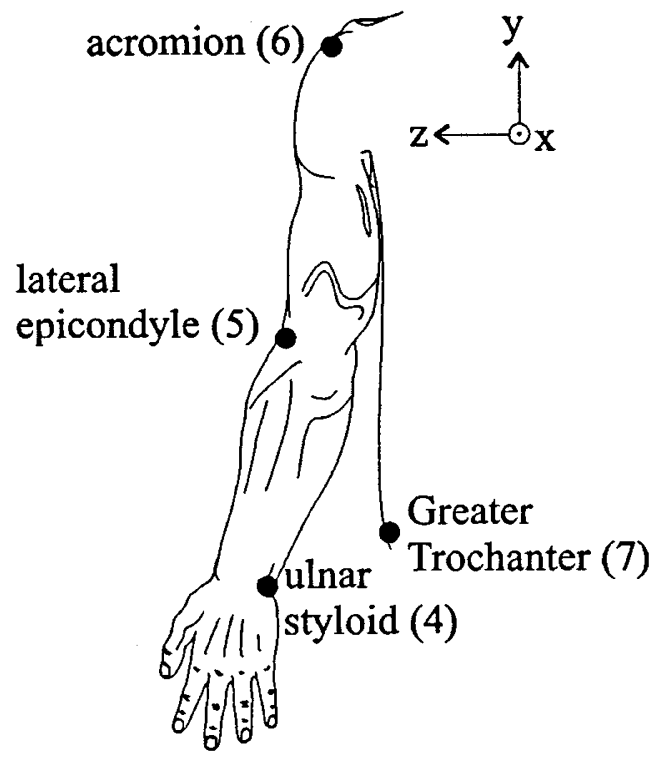

Figure 1 Marker location and number: Location of the four markers used for the kinematic analysis. The numbers after each marker are referenced in the Appendix. In addition, the laboratory local coordinate system is shown in reference to the subject sitting erect 

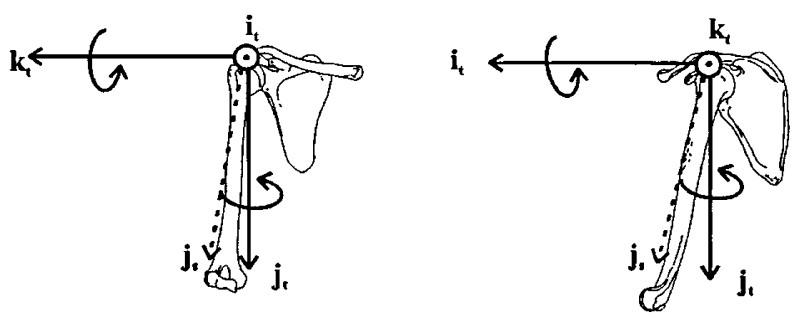

Figure 2 Shoulder local coordinate system: Shoulder motion is described in terms of a coordinate system based on the trunk. The axis $\mathrm{j}_{\mathrm{s}}$ is projected onto this coordinate system to determine the angle of the shoulder with respect to the trunk

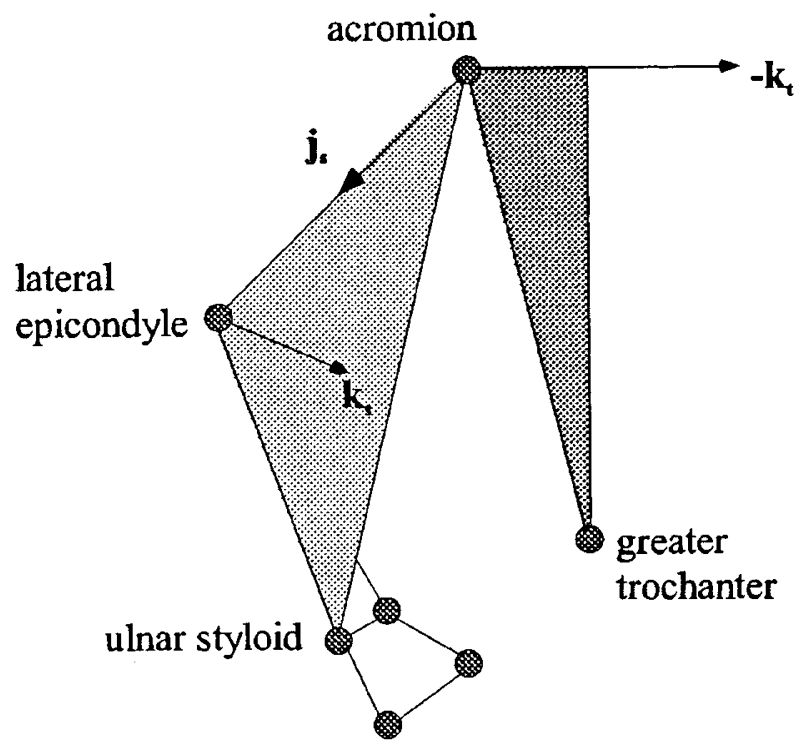

\section{Shoulder plane Trunk plane}

Figure 3 Planes of the shoulder and trunk: Planes formed by the trunk and shoulder local coordinate system are presented. By rotating the shoulder plane such that $\mathrm{j}_{\mathrm{s}}$ and $\mathrm{k}_{\mathrm{t}}$ are co-linear internal and external rotation of the arm can be determined

SMART $^{\text {Wheel }}$ and low-pass filtered at $30 \mathrm{~Hz}^{20}$ For each subject, five propulsive phases and four complete strokes were analyzed.

\section{Data analysis}

\section{Local coordinate system}

Using the marker system shown in Figure 1, it is possible to describe movement in terms of actual shoulder and elbow anatomy. The mathematics used to calculate the kinematics in a local coordinate system are presented in the Appendix. In summary, the arm and trunk markers (see Figure 1) are used to define the axes of a local coordinate system based at the shoulder (see Figures 2 and 3) and elbow (see Figure 4).

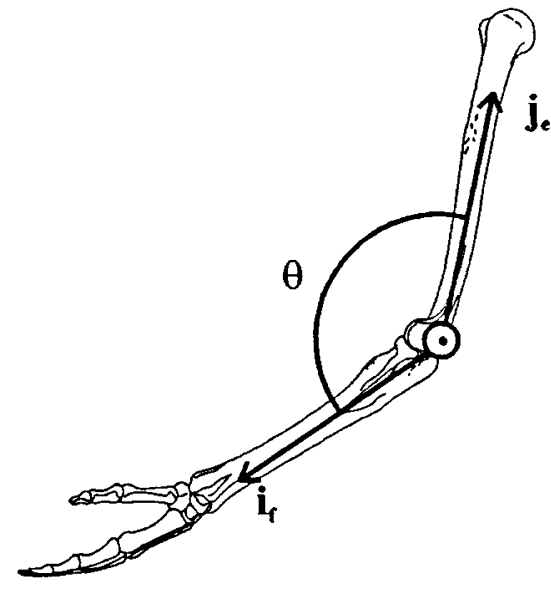

Figure 4 Elbow local coordinate system: The angle between the $i_{\mathrm{f}}$ and $\mathrm{j}_{\mathrm{s}}(\theta)$ is the flexion/extension angle of the elbow

\section{Shoulder coordinate system}

The origin of the shoulder coordinate system is the acromion. The first axis, $\mathrm{k}_{\mathrm{t}}$, is defined as a line parallel to the $\mathrm{z}$ axis of the lab coordinate system and through the acromion. The $\mathrm{z}$ axis is parallel to a line through the axles of the wheelchair wheels and is shown in Figure 1. The second axis, $\mathrm{j}_{\mathrm{t}}$, is perpendicular to the first axis and in the direction of the greater trochanter of the hip. The last axis, $i_{t}$, is perpendicular to the plane formed by $\mathrm{k}_{\mathrm{t}}$ and $\mathrm{j}_{\mathrm{t}}$ (see Figure 2). This coordinate system accounts for trunk flexion and extension during propulsion, but assumes no trunk rotation or lateral bending. The model also assumes that a line connecting the subject's shoulders is parallel to the floor.

By projecting the line between the acromion and the lateral epicondyle, $\mathrm{j}_{\mathrm{s}}$, onto the planes of this coordinate system shoulder flexion/extension can be determined in both the sagittal and horizontal planes and abduction/adduction. Movement about $\mathrm{k}_{\mathrm{t}}$ represents flexion/extension of the shoulder in the sagittal plane. Motion about $i_{t}$ represents abduction/adduction. Movement about the $\mathrm{j}_{\mathrm{t}}$ axis represents flexion/ extension in the horizontal plane.

The determination of internal/external rotation at the shoulder is more complex. In order to determine internal/external rotation, a plane must be defined. This plane is formed by the markers over the acromion, lateral epicondyle, and ulnar styloid (see Figure 3 ). The rotation of this plane in relation to the local coordinate system allows for determination of internal and external rotation. For the shoulder, an angle of $0^{\circ}$ represents the anatomically neutral position of arm.

\section{Elbow coordinate system}

The origin of the elbow coordinate system is the lateral epicondyle. The first axis, $\mathrm{j}_{\mathrm{s}}$, is defined as the line between the lateral epicondyle and the acromion. The 
second axis, $i_{s}$, is perpendicular to the first axis and in the direction of the ulnar styloid. The last axis, $\mathrm{k}_{\mathrm{s}}$, is perpendicular to the plane formed by $\mathrm{i}_{\mathrm{s}}$ and $\mathrm{j}_{\mathrm{s}}$. By projecting the line between the ulnar styloid and the lateral epicondyle, $i_{f}$, into this coordinate system elbow flexion/extension, the primary motion at the elbow, can be determined.

For this study, the onset of propulsion was defined as the point at which a propulsive moment, as measured by the SMART ${ }^{\text {Wheel }}$, was applied to the pushrim. The end of the propulsion and the beginning of recovery were defined as the point at which the propulsive moment at the pushrim returned to zero. From the motion curves peak accelerations as well as minimum and maximum angles during the propulsion phase were obtained. Based on a possible relationship between injury mechanisms and extremes of motion and acceleration under a loaded condition, peak values during the propulsive phase were examined. Finally, the total range of motion (ROM) during the entire stroke was calculated.

\section{Statistics}

All parameters were computed using Matlab. (the MathWorks, Inc., 24 Prime Park Way, Natick, MA 01760). To determine which parameters were reliable across repeated strokes and speeds and, therefore, suitable for further statistical analysis, a two-step approach was used. First, the interstroke reliability was evaluated by intraclass $\mathrm{R}$ correlation coefficients. ${ }^{21} \mathrm{~A}$ parameter was considered reliable and stable if intraclass $\mathrm{R}$ was $>0.60^{22}$ for both speeds. Second, for parameters that met the preceding criteria, Cronbach's coefficient

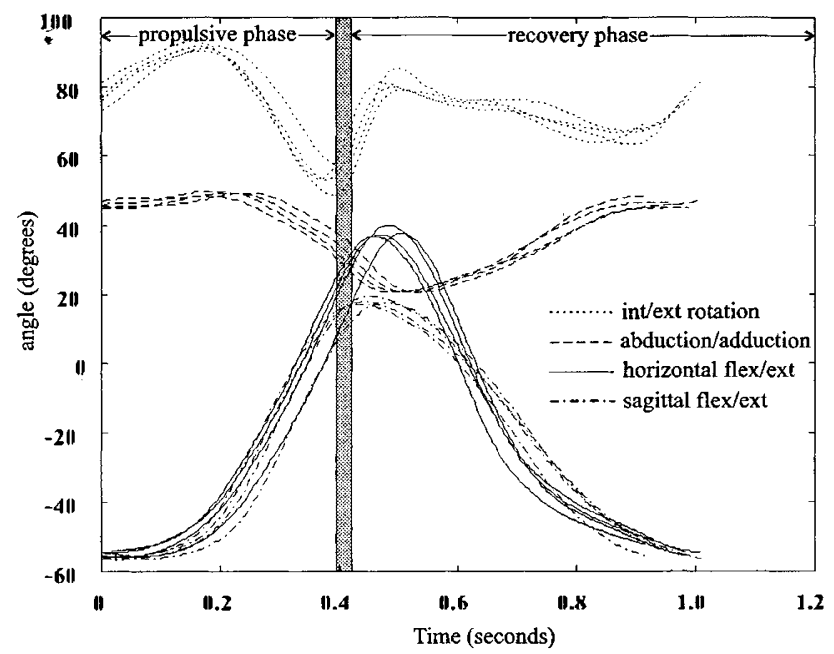

Figure 5 Shoulder movement for a single subject: Four consecutive strokes for a single subject are presented. The beginning of the stroke is at time equals zero seconds. The gray area indicates the time during which the subject went from the propulsive to the recover phase. This area cannot be represented as a line because of differences in propulsion time from stroke to stroke alpha was computed to determine the measurement precision or increased reliability of creating aggregated scores across the repeated strokes. ${ }^{23}$ The summed score was considered to have good reliability if coefficient alpha was $>0.80$ at both speeds. For parameters that met both of these conditions, paired $t$-tests were conducted to test for significant performance differences between the two testing speeds. A $P$ value of less than 0.05 was considered statistically significant. Experimentwise error rate procedures were not used due to small sample size.

\section{Results}

\section{Descriptive}

Figure 5 presents shoulder motion for four propulsive strokes at the same speed for a single subject. This data
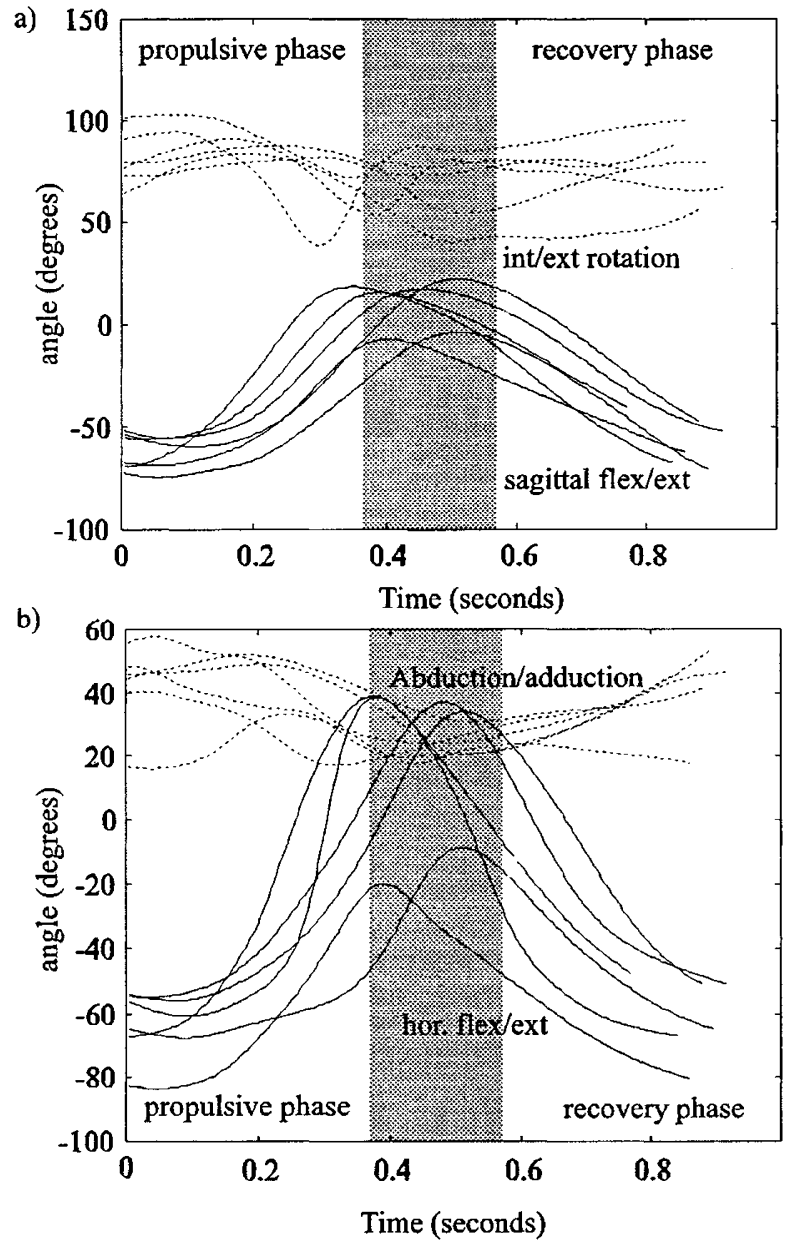

Figure 6 Shoulder movement for all subjects: The mean of four consecutive strokes for each subject is presented. The beginning of the stroke is at time equals zero seconds. The gray area indicates the time during which the subject went from the propulsive to the recover phase. This area is larger than in Figure 5 because it represents the differences in mean propulsion time between subjects 
was plotted to show stroke to stroke variation in a single subject. Figure 6 displays the mean stroke for all six subjects at the slow speed. In general, during the propulsive stroke there is a move towards decreased internal rotation and abduction. In addition the shoulder goes from a position of extension to that of flexion. In each of these Figures, the plot begins at the onset of the stroke as defined above. Differences exist in the duration of the stroke in an individual, thus individual curves are of different length. The gray area in the figures represents the transition from propulsion to recovery.

Figure 7 shows motion at the elbow for two different subjects at two different speeds. During the initial phase of propulsion the elbow goes from an
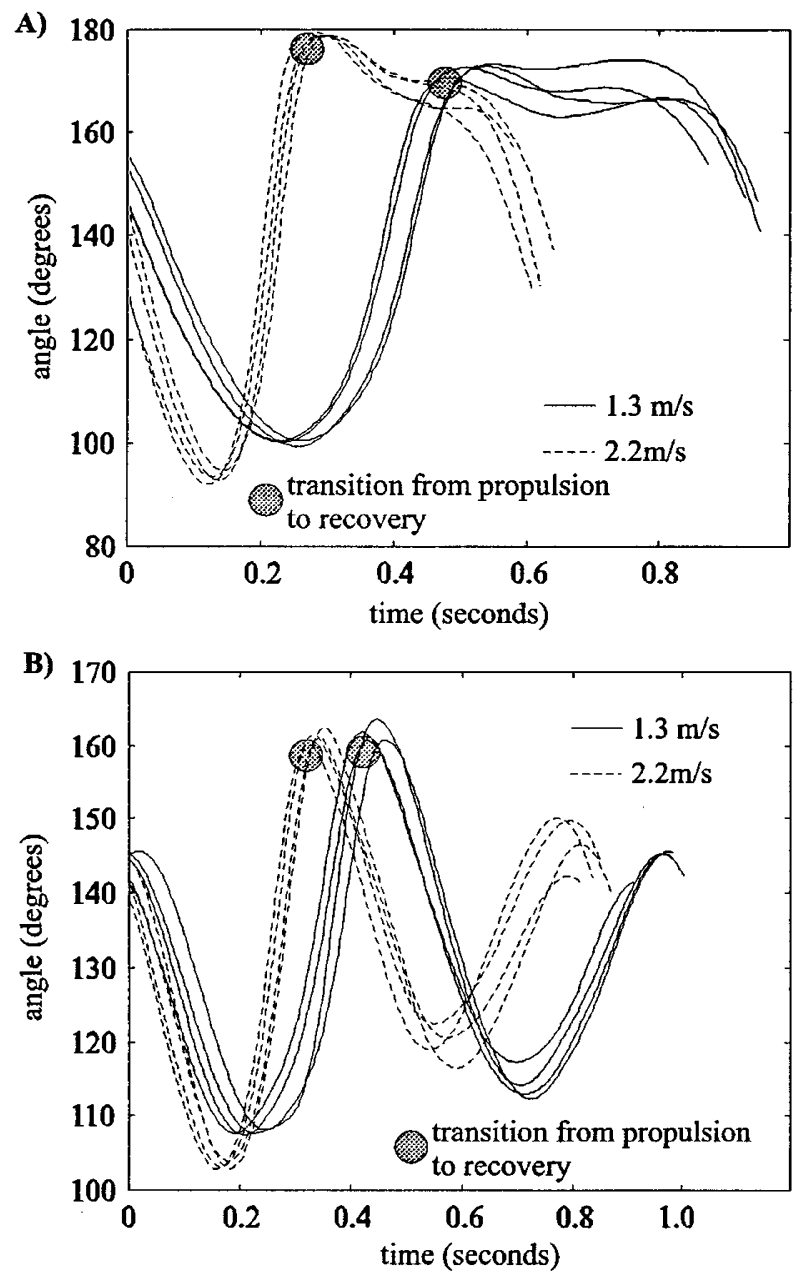

Figure 7 Elbow movement for a single subject at two speeds: Four consecutive strokes for a single subject at two speeds are presented. The beginning of the stroke is at time equals zero seconds. The gray area indicates the time during which the subject went from the propulsive to the recover phase. Two different types of motion during recovery are presented, (a) shows gradual flexion during recovery, (b) shows a more rapid flexion followed by extension during recovery extended position into greater flexion. At approximately the midpoint of propulsion the elbow begins to extend until the end of the stroke. Two distinct patterns were seen during recovery. Half of the subjects gradually flexed the arm during recovery in preparation for the next stroke. This pattern is depicted in Figure 7a. The remainder of the subjects flexed then extended the elbow prior to initiating the next stroke. This is seen in Figure $7 \mathrm{~b}$. For each subject, at both the shoulder and the elbow, the patterns seen during the stroke were consistent across speeds.

\section{Changes with speed}

Table 1 presents the mean maximum and minimum angles during propulsion, the mean total ROM for the entire stroke, and the peak accelerations. All variables in the table met the criteria for stability set forth in the analysis section. During propulsion the minimum abduction angle was found to significantly decrease with speed. In other words, as the subjects propelled the wheelchair faster they held their arms in a more adducted position. For the entire stroke, both sagittal plane flexion/extension and elbow flexion/extension ROM were found to increase with increasing speeds. Sagittal plane flexion/extension ROM increased by approximately $8^{\circ}$ and elbow flexion/extension ROM increased by approximately $4^{\circ}$. This increased ROM with increased speed can be seen in Figure 7. Finally, peak acceleration during propulsion was found to increase in sagittal plane flexion/extension, abduction/ adduction and elbow flexion/extension.

\section{Discussion}

The goal of much of the effort towards investigating wheelchair propulsion biomechanics is injury reduction. In order to gain insight into injuries it is important to be able to describe motions during propulsion in anatomically relevant terms. This paper presents a robust methodology for describing shoulder motion in relation to the trunk through the use of a local coordinate system. Using the local coordinate system we were able to find reliable kinematic measures capable of differentiating between two conditions. Using this same local coordinate system, it will be possible to place measured forces and moments in anatomically relevant terms.

There are inherent difficulties in comparing this work with other studies. The difficulties arise because of differences in data collection, wide variations in mathematical models, and lack of description of metrics presented. A number of studies have evaluated wheelchair propulsion kinematics in two dimensions. $^{7-9}$ In additional studies kinematic data is presented, but it is not the focus of the article, and the methods and/or results do not provide enough information to allow for comparison with our work. $^{10,11,13}$ Table 2 presents a comparison of our 
Table 1 Key kinematic variables

\begin{tabular}{|c|c|c|c|c|c|c|c|c|}
\hline \multirow[b]{2}{*}{ Speed $(\mathrm{m} / \mathrm{s})$} & \multicolumn{2}{|c|}{$\begin{array}{l}\text { Maximum angle on } \\
\text { the pushrim } \\
\left.()^{\circ}\right)\end{array}$} & \multicolumn{2}{|c|}{$\begin{array}{l}\text { Minimum angle on } \\
\text { the pushrim } \\
\left({ }^{\circ}\right)\end{array}$} & \multicolumn{2}{|c|}{$\begin{array}{l}\text { Maximum total } \\
\text { range of motion }\end{array}$} & \multicolumn{2}{|c|}{$\begin{array}{c}\text { Maximum angular } \\
\text { acceleration } \\
\left(\% / s^{2}\right)\end{array}$} \\
\hline & 1.3 & 2.2 & 1.3 & 2.2 & 1.3 & 2.2 & 1.3 & 2.2 \\
\hline Sagittal plane & 6.2 & 11.1 & -64.0 & -68.8 & $74.8 \dagger$ & $82.6 \dagger$ & $4044 \dagger$ & $7146 \dagger$ \\
\hline Flexion/extension & $(16.9)$ & $(13.7)$ & $(8.0)$ & (10.8) & (9.4) & $(8.5)$ & $(946)$ & $(1705)$ \\
\hline Horizontal plane & 11.3 & 22.2 & -64.9 & -66.0 & 86.0 & 96.9 & 8863 & 20875 \\
\hline Flexion/extension & $(28.5)$ & $(25.8)$ & $(10.6)$ & $(10.7)$ & $(19.3)$ & $(28.0)$ & $(5857)$ & $(18456)$ \\
\hline Abduction & 47.0 & 54.2 & $24.5^{*}$ & $21.6^{*}$ & 26.1 & 37.4 & $2678 \dagger$ & $4928 \dagger$ \\
\hline Adduction & (8.9) & $(18.7)$ & (6.7) & $(7.2)$ & $(5.2)$ & $(15.4)$ & (767) & (1311) \\
\hline Internal/external & 90.7 & 92.8 & 55.2 & 51.3 & 37.3 & 44.4 & 9564 & 24588 \\
\hline Rotation & (7.5) & $(9.1)$ & $(16.3)$ & (18.2) & (14.9) & (19.6) & $(6513)$ & $(21108)$ \\
\hline Elbow & 156.2 & 155.7 & 107.7 & 103.3 & $54.0 *$ & $58.1 *$ & $9355^{*}$ & $12889^{*}$ \\
\hline Flexion/extension & $(14.9)$ & $(9.6)$ & $(8.7)$ & $(7.7)$ & $(9.9)$ & (10.4) & $(4120)$ & $(5572)$ \\
\hline
\end{tabular}

Note: all variables were statistically reliable. *Statistical difference between speeds $(P<0.05)$. $\dagger$ Statistical difference between speeds $(P<0.01)$

Table 2

\begin{tabular}{|c|c|c|c|c|}
\hline Study & $\begin{array}{l}\text { Sagittal flexion/ } \\
\text { extension }\left({ }^{\circ}\right)\end{array}$ & $\begin{array}{c}\text { Abduction/ } \\
\left.\text { adduction ( }{ }^{\circ}\right)\end{array}$ & $\begin{array}{l}\text { Internal/external } \\
1 \text { rotation }\left({ }^{\circ}\right)\end{array}$ & $\begin{array}{l}\text { Elbow flexion/ } \\
\text { extension }\left({ }^{\circ}\right)\end{array}$ \\
\hline This study, slow speed & $-64-11$ & $21-47$ & $54-91$ & $102-158$ \\
\hline Bednarczyk et al. ${ }^{11}$ & $-70-20$ & $30-70$ & not presented & $90-140$ \\
\hline Rao et al. ${ }^{13}$ & $-57-23$ & $23-57$ & $11-86$ & $104-146$ \\
\hline
\end{tabular}

study to two other studies looking at shoulder and elbow motion. Only studies that described motion in three dimension in a standard wheelchair and are detailed enough to allow for interpretation of the data are presented.

Bednarczyk et al studied 10 adults and 10 children pushing on a track. ${ }^{12}$ They used Peak technologies software to calculate shoulder and elbow motion. In Bednarczyk's study, internal/external rotation angles were not calculated. In addition, no statistics testing the reliability of measures were performed. Finally the mathematics behind determining the kinematic parameters were not presented. Rao et al used a local coordinate system to describe upper extremity motion in 16 male wheelchair users. ${ }^{14}$ This study used a novel approach to determine internal and external rotation, clearly defined the mathematical models, and examined statistical reliability. Unlike our study, shoulder motion was referenced to a laboratory coordinate system, not trunk motion. Referencing shoulder motion to a laboratory system means that trunk flexion and extension will confound shoulder motion measures. Trunk range of motion has been shown to be as high as $15^{\circ} .{ }^{12,13}$ This difference in mathematical models may explain the differences seen internal/ external rotation and flexion/extension motion. Finally, the parameters described in the Rao study were not tested for their ability to differentiate between two different conditions.

We chose to investigate end ROM while on the pushrim, peak accelerations, and total ROM for a number of reasons. End ROM while on the pushrim was investigated because the arm would likely be most prone to injury while externally loaded (pushing on the pushrim) and at extremes of range. Peak accelerations were investigated because during maximum acceleration the forces caused by inertia of the limb are the largest. These higher forces may also be related to upper extremity injuries. Finally, a large ROM during the entire stroke may also be associated with injuries.

Future research is needed to investigate if a link can be found between biomechanical measures and the development of upper extremity injuries. It is reassuring that the measures analyzed in this paper were found to be reliable and changes were seen with speed. Once a link can be established between injury and propulsion biomechanics, it may be possible to minimize injurious mechanics thus leading to a reduction in the risk of injury.

\section{References}

1 Pentland WE, Twomey LT. The weight-bearing upper extremity in women with long term paraplegia. Paraplegia 1991; 29: 521 530 .

2 Gellman H, Sie I, Waters RL. Late complications of the weightbearing upper extremity in the paraplegic patient. Clin Orthop Relat $R$ 1988; 233: $132-135$

3 Nichols PJ, Norman PA, Ennis JR. Wheelchair user's shoulder? Shoulder pain in patients with spinal cord lesions. Scand $J$ Rehabil Med 1979; 11: 29-32. 
4 Sie IH, Waters RL, Adkins RH, Gellman H. Upper extremity pain in the postrehabilitation spinal cord injured patient. Arch Phys Med Rehabil 1992; 73: 44-48.

5 Bayley JC, Cochran TP, Sledge CB. The weight-bearing shoulder. The impingement syndrome in paraplegics. J Bone Joint Surg [Am] 1987; 69: 676-678.

6 Wylie EJ, Chakera TM. Degenerative joint abnormalities in patients with paraplegia of duration greater than 20 years. Paraplegia 1988; 26: 101 - 106.

7 Sanderson DJ, Sommer HJ. Kinematic features of wheelchair propulsion. J Biomech 1985; 18: $423-429$.

8 Wang YT et al. Three-dimensional kinematics of wheelchair propulsion across racing speeds. Adapted Physical Activity Qtrly 1995; 12: $78-89$

9 van der Woude LHV, Veeger DJ, Rozendal RH, Sargeant TJ. Seat height in handrim wheelchair propulsion. J Rehabil Res Dev 1989; 26: $31-50$.

10 Hughes CJ, Weimar WH, Sheth PN, Brubaker CE. Biomechanics of wheelchair propulsion as a function of seat position and userto-chair interface. Arch Phys Med Rehabil 1992; 73: 263-269.

11 Rodgers MM et al. Biomechanics of wheelchair propulsion during fatigue. Arch Phys Med Rehabil 1994; 75: 85-93.

12 Bednarczyk JH, Sanderson DJ. Kinematics of wheelchair propulsion in adults and children with spinal cord injury. Arch Phys Med Rehabil 1994; 75: 1327 - 1334.

13 Vanlandewijck YC, Spaepen AJ, Lysens RJ. Wheelchair propulsion efficiency: Movement pattern adaptation to speed changes. Med Sci Sports Exerc 1994; 26: 1373-1381.

14 Rao SS et al. Three-dimensional kinematics of wheelchair propulsion. IEEE Trans Rehab Engr 1996; 4: 152-160.

15 Asato KT, Cooper RA, Robertson RN, Ster JF. SMARTWheels: Development and testing of a system for measuring manual wheelchair propulsion dynamics. IEEE Trans Biomed Eng 1993; 40: $1320-1324$.

16 VanSickle DP et al. Single Point Center of Pressure for Wheelchair Propulsion. (1996) Proceedings RESNA '96 Exploring New Horizons - Pioneering the 21st Century, Salt Lake City, UT, pp $498-500$.

17 Cooper RA et al. A Method for Analyzing Center of Pressure During Manual Wheelchair Propulsion. IEEE Trans Rehab Engr 1995; 3: $289-298$.

18 Vosse AJ, Cooper RA, Dhaliwal B. Computer control of a wheelchair dynamometer. (1990) Proceedings 13th Annual RESNA Conference, Washington, DC, pp 59-60.

19 Cooper RA. A force/energy optimization model for wheelchair athletics. IEEE Trans Syst Man Cybern 1990; 20: $444-449$.

20 DiGiovine CP et al. Frequency Domain Analysis of Wheelchair Pushrim Forces and Moments. (1996) Proceedings of the RESNA 96' Annual Conference, Arlington, VA, 16: pp 238-240.

21 Bartko JJ. On Various Intraclass Correlation Reliability Coefficients. Psychol Bull 1976; ??: $762-765$.

22 Cicchetti DV, Sparrow SA. Developing criteria for establishing interrater reliability of specific items: applications to assessment of adaptive behavior. Am J Mental Deficiency 1981; 86: 127-137.

23 Fleiss JL. The design and analysis of clinical experiments, New York, Wiley; 1986.

\section{Appendix}

Calculation of shoulder angles forces and moments in a local coordinate system

A body oriented coordinate system is required to determine anatomical shoulder motion. This is accomplished with set of axes attached to the trunk. The trunk coordinate system is illustrated in Figures 2 and 3 . The first axis $k_{t}$ is defined as line passing through the acromion and parallel to the $\mathrm{z}$ axis of the laboratory coordinate system shown in Figure 1. This model assumes the only motion occurring at the trunk is flexion and extension. Rotation about the body's midline and lateral bending are ignored. The $i_{t}$ axis is defined as the cross product of $\mathrm{k}_{\mathrm{t}}$ axis and the unit vector from the acromion to the greater trochanter. Finally, the $\mathrm{j}_{\mathrm{t}}$ axis is the cross product of $\mathrm{i}_{\mathrm{t}}$ and $\mathrm{k}_{\mathrm{t}}$.

$$
\begin{aligned}
\mathbf{K}_{t} & =\text { laboratory } \mathrm{z} \text { axis } \\
\mathbf{i}_{t} & =k_{t} \times \frac{\mathbf{v}_{6-7}}{\left\|\mathbf{v}_{6-7}\right\|} \\
\mathbf{j}_{t} & =\mathbf{i}_{t} \times \mathbf{k}_{t}
\end{aligned}
$$

The unit local coordinate system is then used to define a rotation matrix between the world and trunk coordinate systems.

$$
\mathbf{R}_{W t}=\left[\begin{array}{lll}
i_{t x} & i_{t y} & i_{t z} \\
j_{t x} & j_{t y} & j_{t z} \\
k_{t x} & k_{t y} & k_{t z}
\end{array}\right] \mathbf{R}_{t W}=\mathbf{R}_{W t}
$$

Based upon these definitions, the motion at the shoulder in local coordinates can be determined.

In this model, the humerus is defined by a line connecting the acromion process marker and the lateral epicondyle marker. Using the rotation matrix, the vector between these markers can be placed into the trunk coordinate system:

$$
\left[\begin{array}{c}
{ }^{t} \mathbf{v}_{6-5, x} \\
{ }^{t} \mathbf{v}_{6-5, y} \\
{ }^{t} \mathbf{v}_{6-5, z}
\end{array}\right]=\mathbf{R}_{W t}\left(\left[\begin{array}{l}
\mathbf{v}_{0-5, x} \\
\mathbf{v}_{0-5, y} \\
\mathbf{v}_{0-5, z}
\end{array}\right]-\left[\begin{array}{l}
\mathbf{v}_{0-6, x} \\
\mathbf{v}_{0-6, y} \\
\mathbf{v}_{0-6, z}
\end{array}\right]\right)
$$

The normalized vector between markers 6 and 5 in the trunk coordinate reference frame is then converted to a unit vector.

$$
\frac{{ }^{t} \mathbf{v}_{6-5}}{\left\|\mathbf{v}_{6-5}\right\|} \equiv\left[\begin{array}{lll}
a & b & c
\end{array}\right]
$$

Each term on the right side of the equation is a component of the unit vector on the left side of the equation. Since each term is known it is possible to determine specific angles:

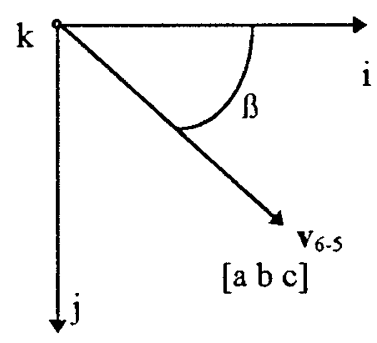

This figure is oriented looking along the $\mathrm{k}$ axis which travels between the left and right shoulder, $\mathrm{j}$ travels down toward the hip. $\mathrm{V}_{6-5}$ is the unit vector from 6 to 5 along the humerus. Therefore $\beta$ is the flexion extension angle and when $\beta=0^{\circ}$ the arm is flexed forward to $90^{\circ}$.

$$
\beta=\arctan (\mathrm{b} / \mathrm{a})
$$


Defining flexion and extension at the arm as being $0^{\circ}$ when the arm is in an anatomically neutral position $90^{\circ}$ must be subtracted from $\beta$.

To determine abduction and adduction, it is necessary to look down the $i$ axis which is perpendicular to the plane formed by the chest wall:

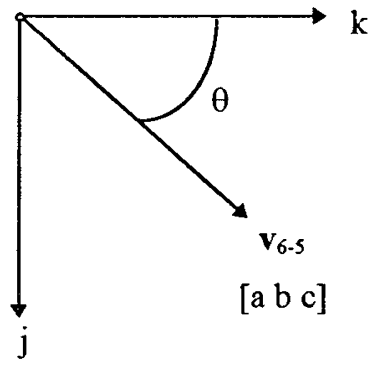

$$
\theta=\arctan (\mathrm{b} / \mathrm{c})
$$

When $\theta=0^{\circ}$ the arm is abducted to $90^{\circ}$. In order to define anatomically neutral as $0^{\circ}, 90^{\circ}$ is added to $\theta$.

Determining internal and external rotation is more complex. Using a line to define the humerus does not account for rotation. Therefore, a plane connecting the acromion process, the lateral epicondyle, and the ulnar styloid is used to describe rotation of the humerus. This plane is labeled the shoulder plane in Figure 4. The shoulder coordinate system is defined by the triple $\left(i_{s}, j_{s}, k_{s}\right)$. These unit basis vectors are defined as:

$$
\mathbf{J}_{s}=\frac{\mathbf{v}_{6-5}}{\left\|\mathbf{v}_{6-5}\right\|} \quad \mathbf{k}_{s}=\frac{\mathbf{v}_{5-4}}{\left\|\mathbf{v}_{5-4}\right\|} \times \frac{\mathbf{v}_{6-5}}{\left\|\mathbf{v}_{6-5}\right\|} \quad \mathbf{i}_{s}=\mathbf{k}_{s} \times \mathbf{j}_{s}
$$

In order to determine internal and external rotation $\mathrm{j}_{\mathrm{s}}$, must be placed in a trunk coordinate system. Then the angles necessary to rotate the $\mathrm{j}_{\mathrm{s}}$ axis such that it is parallel the $\mathrm{k}_{\mathrm{t}}$ are determined:

$$
\begin{aligned}
J_{s t} & =j_{s} \mathbf{R}_{W t} \\
\operatorname{rot} 1 & =\left(-\arctan \left(j_{s t}(\mathrm{~b}) / j_{s t}(\mathrm{a})\right)-\pi / 2\right) \\
\operatorname{rot} 2 & =\left(-\arctan \left(j_{s t}(\mathrm{c}) / j_{s t}(\mathrm{~b})\right)-\pi / 2\right)
\end{aligned}
$$

The first rotation angle (rot 1 ) is about $\mathrm{k}_{\mathrm{t}}$ the second rotation angle is about $i_{t}, k_{s}$ is then projected into the trunk coordinate system and rotated through the two angles:

$$
\begin{gathered}
\mathbf{k}_{s}=\mathbf{k}_{s} \mathbf{R}_{W t} \\
\mathrm{k}_{s t r 1}=\mathrm{k}_{s t}=\left[\begin{array}{ccc}
\cos (\operatorname{rot} 1) & -\sin (\operatorname{rot} 1) & 0 \\
\sin (\operatorname{rot} 1) & \cos (\operatorname{rot} 1) & 0 \\
0 & 0 & 1
\end{array}\right] \\
\mathrm{k}_{s t r}=\mathrm{k}_{s t r 1}=\left[\begin{array}{ccc}
1 & 0 & 0 \\
0 & \cos (\operatorname{rot} 2) & -\sin (\operatorname{rot} 2) \\
0 & \sin (\operatorname{rot} 2) & \cos (\operatorname{rot} 2)
\end{array}\right]
\end{gathered}
$$

$\mathbf{k}_{\text {str }}$ is then used to determine the internal/external rotation angle:

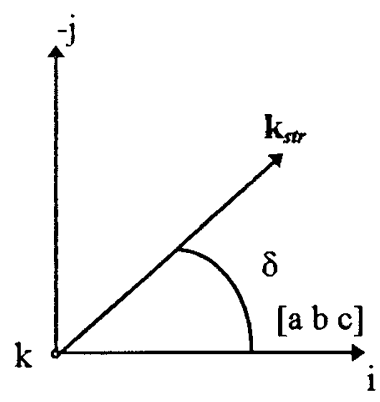

$$
\delta-\arctan \left(\mathrm{k}_{s}(\mathrm{~b}) / \mathrm{k}_{\text {str }}(\mathrm{a})\right)
$$

When the shoulder is in a neutral position, $\mathrm{k}_{\text {str }}$ is pointing straight up and $\delta=90^{\circ}$. Therefore $90^{\circ}$ must be subtracted from $\delta$ to have a neutral position recorded as $0^{\circ}$.

\section{Calculation of elbow angles using a local coordinate} system

In order to place the elbow into a local coordinate system, a shoulder local coordinate system must be defined. The shoulder coordinate system consist of three unit vectors; $\mathrm{i}_{\mathrm{s}}, \mathrm{j}_{\mathrm{s}}$, and $\mathrm{k}_{\mathrm{s}}$. The unit vector, $\mathrm{j}_{\mathrm{s}}$, lies along the humerus of the subject. This is defined by:

$$
j_{s}=\frac{\mathbf{v}_{6-5}}{\left\|\mathbf{v}_{6-5}\right\|}
$$

The unit vector, $\mathrm{k}_{\mathrm{s}}$, is defined as the vector perpendicular to the plane that is formed by the humerus and forearm. The unit vector, $\mathrm{k}_{\mathrm{s}}$, calculated by the cross-product of the unit vector, $\mathrm{j}_{\mathrm{s}}$, and a unit vector lying along the forearm segment. The final unit vector, $i_{s}$, is defined by the cross-product of $j_{s}$ and $\mathrm{k}_{\mathrm{s}}$ :

$$
k_{s}=j_{s} \times \frac{\mathbf{v}_{4-5}}{\left\|\mathbf{v}_{4-5}\right\|}, \quad i_{s}=j_{s} \times k_{s} .
$$

This results in a rotation matrix from the laboratory coordinate system to a shoulder coordinate system:

$$
R_{L S}=\left[\begin{array}{lll}
i_{s, x} & i_{s, y} & i_{s, z} \\
j_{s, x} & j_{s, y} & j_{s, z} \\
k_{s, x} & k_{s, y} & k_{s, z}
\end{array}\right]
$$

Anatomical elbow flexion and extension angles can be calculated by rotating the ulna, defined by the unit vector, $i_{\mathrm{e}}$, into the shoulder coordinate system,

$$
\begin{gathered}
i_{f}=\frac{\mathbf{v}_{4-5}}{\left\|\mathbf{v}_{4-5}\right\|} \\
U_{s}=R_{L S} \times\left[i_{f}\right]^{T}
\end{gathered}
$$

resulting in: 


$$
U s=\left[i_{f_{-} L S}, j_{f_{-} L S}, k_{f_{-} L S}\right] .
$$

Elbow flexion/extension is described by:

$$
\theta=\arctan \left(i_{f-L S} / j_{f_{L L S}}\right)
$$

The elbow is in full extension when $\theta=180^{\circ}$. 